\title{
Video Article \\ A New Single Chamber Implantable Defibrillator with Atrial Sensing: A Practical Demonstration of Sensing and Ease of Implantation
}

\author{
Dietmar Bänsch ${ }^{1}$, Ralph Schneider ${ }^{1}$, Ibrahim Akin $^{1}$, Cristoph A. Nienaber ${ }^{1}$ \\ ${ }^{1}$ Heart Center Rostock, University Hospital of Rostock, Germany \\ Correspondence to: Dietmar Bänsch at dietmar.baensch@med.uni-rostock.de \\ URL: https://www.jove.com/video/3750 \\ DOI: doi:10.3791/3750
}

Keywords: Medicine, Issue 60, Implantable defibrillator, dual chamber, single chamber, tachycardia detection

Date Published: 2/28/2012

Citation: Bänsch, D., Schneider, R., Akin, I., Nienaber, C.A. A New Single Chamber Implantable Defibrillator with Atrial Sensing: A Practical Demonstration of Sensing and Ease of Implantation. J. Vis. Exp. (60), e3750, doi:10.3791/3750 (2012).

\section{Abstract}

Implantable cardioverter-defibrillators (ICDs) terminate ventricular tachycardia (VT) and ventricular fibrillation (VF) with high efficacy and can protect patients from sudden cardiac death (SCD). However, inappropriate shocks may occur if tachycardias are misdiagnosed. Inappropriate shocks are harmful and impair patient quality of life. The risk of inappropriate therapy increases with lower detection rates programmed in the ICD. Single-chamber detection poses greater risks for misdiagnosis when compared with dual-chamber devices that have the benefit of additional atrial information. However, using a dual-chamber device merely for the sake of detection is generally not accepted, since the risks associated with the second electrode may outweigh the benefits of detection. Therefore, BIOTRONIK developed a ventricular lead called the

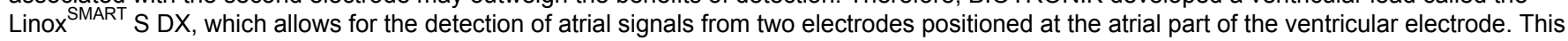
device contains two ring electrodes; one that contacts the atrial wall at the junction of the superior vena cava (SVC) and one positioned at the free floating part of the electrode in the atrium. The excellent signal quality can only be achieved by a special filter setting in the ICD (Lumax 540 and 740 VR-T DX, BIOTRONIK). Here, the ease of implantation of the system will be demonstrated.

\section{Video Link}

The video component of this article can be found at https://www.jove.com/video/3750/

\section{Protocol}

\section{Introduction}

Implantable cardioverter-defibrillators (ICD) prevent sudden cardiac death (SCD) in patients after survived SCD or at risk of SCD due to impaired left ventricular function. However, the danger of inappropriate therapies is high, particularly in single-chamber devices. These superfluous electrical discharges can be harmful and impair patient quality of life. Atrial detection may improve device performance and reduce the risk of inappropriate shock. ${ }^{1,2}$ However atrial detection requires an atrial electrode which in itself carries a significant associated risk. ${ }^{3}$ One manufacturer, BIOTRONIK, developed a ventricular single chamber electrode with atrial sensing capabilities. The first electrode was the Kainox $\mathrm{A}+$, which revealed excellent sensing capabilities. ${ }^{4}$ However, it was not commonly used due to electrode stiffness and large diameter ( $3.5 \mathrm{~mm}$, $10.5 \mathrm{~F})$. Further developments of this electrode lead to the creation of the Linox ${ }^{\text {smart }} \mathrm{S} \mathrm{DX}$ lead, which has a smaller diameter $(2.6 \mathrm{~mm}, 7.8 \mathrm{~F})$ and demonstrates excellent implantation features and sensing capabilities. ${ }^{5}$ This paper depicts the placement of this electrode, in order to highlight the data from the master study and to demonstrate both the ease of implantation and the excellent sensing capabilities of this electrode.

\section{Case Presentation}

1. The patient was a 40 year-old female smoker with a history of coronary artery disease (CAD), recurrent anterior myocardial infarction (AMI) status-post percutaneous coronary intervention $(\mathrm{PCl})$, impaired left ventricular function (LVEF= 25\%), hypercholesterolemia and paroxysmal atrial fibrillation (PAF).

2. Four months ago, medical therapy for heart failure was initiated. Her regimen included beta-blockers, ACE-inhibitors and diuretics; however there was no improvement in left ventricular function.

3. Due to her significantly impaired left ventricular function, the patient was at considerable risk of sudden cardiac death (SCD).

4. On presentation, the patient was in sinus rhythm with loss of R-waves in the chest leads. A 24-hour holter monitor revealed a 32-minute episode of atrial fibrillation.

5. The decision was made to implant a single-chamber ICD with atrial detection capability in order to monitor further episodes of atrial fibrillation and to avoid inappropriate therapy in an otherwise very active patient. 


\section{Diagnosis, Assessment, and Plan}

1. The treatment plan was to implant a Lumax VR-T DX defibrillator with a BIOTRONIK Linox ${ }^{\text {smart }} S$ DX lead under regional anesthesia in the electrophysiological laboratory.

2. A transthoracic echo was performed prior to implantation in order to re-evaluate left ventricular ejection fraction and to exclude a ventricular thrombus, which might prohibit defibrillator testing during implantation.

\section{Implantation Procedure}

1. Peri-procedural preparation included a single intravenous dose of prophylactic antibiotic and subcutaneous heparin. Antithrombotic therapy was maintained because the risk of a coronary event outweighed the risk of bleeding during the procedure

2. The patient was appropriately prepared and draped in a sterile manner.

3. The patient was placed under moderate sedation, with appropriate monitoring including continuous electrical cardiac monitoring, continuous pulse oximetry, and non-invasive blood pressure monitoring.

4. Sedation administered consisted of IV midazolam, fentanyl and propofol along with judicious application of topical anesthesia.

5. The implantation began with puncture of the left subclavian vein through the intact skin and placement of a wire in the inferior vena cava (IVC) . The position was confirmed by X-ray. This unusual approach was used since most interventional electrophysiologists are more experienced in this transcutaneous approach, rather than the more lateral approach of puncturing the vein from the pocket.

6. The skin was cut with a scalpel about $2 \mathrm{~cm}$ below and parallel to the clavicles. After the pocket for the device was prepared below the left clavicle, the wire in the vein was drawn to the pocket and an 8 French sheath introduced with the Seldinger technique.

7. The electrode was then introduced into the SVC via the sheath to the right atrium. After a curved stylet (S 65-K, Biotronik) was introduced into the electrode, the electrode was pushed though the tricuspid valve to the right ventricular outflow tract.

8. A straight stylet (S 65-C, Biotronik) was then introduced into the electrode. The electrode fell to the inferior right ventricle and was maneuvered to the right ventricular apex. The position of the electrode tip as well as general position of the two electrodes in the atrium was monitored by X-ray. The two atrial ring electrodes were placed at the junction of the SVC and right atrium.

9. After the electrode was appropriately positioned, measurements of the atrial and ventricular signals were performed.

10. The pacing threshold for the ventricular electrode was measured (Time in Video = "7:27"). The atrial signal was measured between 6.5 and $8 \mathrm{mv}$ (Time in Video = "7:56"). The atrial signal was enhanced three times with the filter setting in the device. The ventricular signal was measured between 8.4 and $9.5 \mathrm{mV}$. With these sensing and pacing-thresholds, the electrode was fixed at the entrance of the subclavian vein.

11. The electrode was then connected to the defibrillator.

12. All measures were rechecked and ventricular fibrillation was induced by T-wave shock via the defibrillator and automatically terminated by the device. (Time in Video = "11:01")

13. The patient was brought to the ward and remained in the hospital until the following day. On post-operative day 1 , an X-ray was taken to confirm the position of the lead and to exclude a pneumothorax. Sensing and pacing thresholds were rechecked and were similar to intraoperative measurements. The patient was observed for approximately 4 hours in recovery and was also monitored after implantation. Overall, the implantation was successful and the patient was discharged from the hospital the following day. The first three-months of followup were eventless and sensing signals remained stable over this timeframe.

\section{Discussion}

Implantation of the new Linox ${ }^{\text {SMART }}$ S DX electrode along with the Lumax DX ICD, Biotronik, was as easy as the placement of any single chamber ICD. This experience is supported by data from the unpublished master DX-trial. ${ }^{5}$ The Linox ${ }^{\text {SMART }} \mathrm{S}$ DX electrode offers the advantage of atrial sensing without the disadvantage of an atrial lead. The atrial signal was stable over time in our patient as has been demonstrated in the DX-trial. ${ }^{5}$

Dual-chamber devices have demonstrated superiority over single-chamber detection in only one prospective randomized trial, called the $1+1$ trial. ${ }^{1}$ In this trial, patients with slow VT were implanted with a dual-chamber ICD that was either programmed to dual- or single- chamber detection. The combined endpoint was the number of inappropriate therapies in response to SVTs and VTs below the detection rate. For this endpoint, the 1+1-trial demonstrated the significant superiority of dual-chamber (DCH) detection with a long tachycardia detection interval (TDI) compared with conventionally programmed single-chamber $(\mathrm{SCH})$ detection. This finding was revisited by Deisenhofer et al., who randomly assigned patients to $\mathrm{DCH}$ - or SCH ICD implantation. However, their trial did not particularly address patients with slow VTs as the mean tachycardia detection interval was $368 \mathrm{~ms}$, and concluded that single- and dual-chamber devices are equally effective for therapy in lifethreatening ventricular tachyarrhythmias. DCH devices were shown have superior rhythm classification but were no different than SCH devices in terms of minimizing inappropriate therapies during SVTs. ${ }^{6}$ Despite the advantages of a dual chamber ICD for diagnosis and differentiation of tachycardias, the concept of implanting it merely for the sake of detection is generally not accepted, because the improved detection is counterbalanced by the increased risk of lead complications, as well as increased mortality and morbidity. ${ }^{2,7,8}$ Therefore, improvement of implantation technique or a VDD-system for dual chamber detection is necessary. ${ }^{9,10}$. The Linox ${ }^{\text {SMART }}$ S DX electrode combined with the Lumax DX ICD offers this advantage. The sensing facilities are similar to a dual chamber devices and the complication rate is as low as in conventional single chamber ICDs. ${ }^{5}$

\section{Conclusion}

The Lumax VR-T DX has been proven to reliably sense atrial signals and gather valuable information to allow for early detection of atrial arrhythmias. Furthermore, the device offers AV discrimination with SMART for safe shock reduction. The available atrial IEGM channel diagnoses the appropriateness of ICD detection and therapy, thereby reducing inappropriate shocks and gaining information to optimize device programming. 


\section{Disclosures}

Production and Free Access of this video-article is sponsored by BIOTRONIK.

\section{References}

1. Bänsch, D., Steffgen, F., Gronefeld, G., et al. The 1+1 trial - A prospective trial of a dual- versus a single-chamber implantable defibrillator in patients with slow ventricular tachycardias. Circulation. 110, 1022-1029 (2004).

2. Gilliam, F.R., Hayes, D.L., Boehmer, J.P., et al. Real World Evaluation of Dual-Zone ICD and CRT-D Programming Compared to Single-Zone Programming: The ALTITUDE REDUCES Study. Journal of Cardiovascular Electrophysiology. 22, 1023-1029 (2011).

3. Dewland, T.A., Pellegrini, C.N., Wang, Y.F., et al. Dual-Chamber Implantable Cardioverter-Defibrillator Selection Is Associated With Increased Complication Rates and Mortality Among Patients Enrolled in the NCDR Implantable Cardioverter-Defibrillator Registry. Journal of the American College of Cardiology. 58, 1007-1013 (2011).

4. Sticherling, C., Zabel, M., Spencker, S., et al. Comparison of a Novel, Single-Lead Atrial Sensing System With a Dual-Chamber Implantable Cardioverter-Defibrillator System in Patients Without Antibradycardia Pacing Indications Results of a Randomized Study. CirculationArrhythmia and Electrophysiology. 4, 56-63 (2011).

5. Safak E. Assessment of atrial sensing quality in ICD patients with a floating atrial dipole ICD lead (interim results). Europace., 1027 (2011).

6. Deisenhofer, I., Kolb, C., Ndrepepa, G., et al. Do current dual chamber cardioverter defibrillators have advantages over conventional single chamber cardioverter defibrillators in reducing inappropriate therapies? A randomized, prospective study. J. Cardiovasc. Electrophysiol. 12, 134-142 (2001).

7. Dewland, T.A., Pellegrini, C.N., Wang, Y.F., et al. Dual-Chamber Implantable Cardioverter-Defibrillator Selection Is Associated With Increased Complication Rates and Mortality Among Patients Enrolled in the NCDR Implantable Cardioverter-Defibrillator Registry. Journal of the American College of Cardiology. 58, 1007-1013 (2011).

8. Poole, J.E., Gleva, M.J., Mela, T., et al. Complication Rates Associated With Pacemaker or Implantable Cardioverter-Defibrillator Generator Replacements and Upgrade Procedures Results From the REPLACE Registry. Circulation. 122, 1553-1U43 (2010).

9. Niehaus, M., Schuchert, A., Thamasett, S., et al. Multicenter experiences with a single lead electrode for dual chamber ICD systems. Pacing Clin. Electrophysiol. 24, 1489-1493 (2001).

10. Gradaus, R., Block, M., Dorszewski, A., et al. Implantation of a dual chamber pacing and sensing single pass defibrillation lead. Pacing Clin. Electrophysiol. 24, 416-423 (2001). 